\title{
Observation of the Conduction Electron Spin Polarization in the Ag Spacer of a Fe/Ag/Fe Trilayer
}

\author{
H. Luetkens, ${ }^{1,2, *}$ J. Korecki, ${ }^{3,4}$ E. Morenzoni, ${ }^{2}$ T. Prokscha,${ }^{2}$ M. Birke, ${ }^{1}$ H. Glückler, ${ }^{2, \dagger}$ R. Khasanov, ${ }^{2,5}$ H.-H. Klauss, ${ }^{1}$ \\ T. Ślezak,${ }^{3}$ A. Suter, ${ }^{2}$ E. M. Forgan, ${ }^{6}$ Ch. Niedermayer, ${ }^{7}$ and F. J. Litterst ${ }^{1}$ \\ ${ }^{1}$ Institut für Metallphysik und Nukleare Festkörperphysik, TU Braunschweig, D-38106 Braunschweig, Germany \\ ${ }^{2}$ Paul Scherrer Institut, Labor für Myonspin-Spektroskopie, CH-5232 Villigen PSI, Switzerland \\ ${ }^{3}$ Faculty of Physics and Nuclear Techniques, Academy of Mining and Metallurgy, PL-30-059 Kraków, Poland \\ ${ }^{4}$ Institute of Catalysis and Surface Chemistry, Polish Academy of Sciences, PL-30-059 Kraków, Poland \\ ${ }^{5}$ Physik Institut der Universität Zürich, CH-8057 Zürich, Switzerland \\ ${ }^{6}$ School of Physics and Astronomy, University of Birmingham, Birmingham B15 2TT, United Kingdom \\ ${ }^{7}$ Fakultät für Physik, Universität Konstanz, D-78457 Konstanz, Germany
}

(Received 17 December 2002; published 3 July 2003)

The spatially oscillating electron spin polarization in the Ag spacer of a $4 \mathrm{~nm} \mathrm{Fe} / 20 \mathrm{~nm}$ $\mathrm{Ag} / 4 \mathrm{~nm} \mathrm{Fe}(001)$ epitaxial trilayer has been determined by means of low energy muon spin rotation. It oscillates with the same period as the interlayer exchange coupling, but shows a much weaker attenuation at large distances $x$ from the interface. The measured magnetization profile from the inner $14 \mathrm{~nm}$ of the spacer is described by an oscillating polarization decaying as $x^{-0.8(1)}$. This unusual behavior may arise from a full confinement of electron states within the spacer.

DOI: 10.1103/PhysRevLett.91.017204

The fascinating properties of magnetic multilayers have stimulated intense research into both fundamental physics and technical applications of these materials. The discovery, by Parkin et al. [1], that the interlayer exchange coupling (IEC) alternates between ferromagnetic and antiferromagnetic across nonmagnetic metallic spacer layers as a function of spacer thickness has led to increased interest. Much experimental effort has been devoted to measure precisely the strength and oscillation period of the IEC in various systems, and the various theoretical approaches to this phenomenon are able to give a good account of the experimental results (for a recent review, see [2]).

The most prominent approaches to describe the IEC are the Ruderman-Kittel-Kasuya-Yosida (RKKY) [3-5] and quantum-well (QW) models [6-8], which are based on the response of the conduction electrons of the spacer material to the magnetic layers. The RKKY models are adopted from those describing the indirect exchange coupling of magnetic impurities in metals, whereas in the QW models a spin-dependent confinement of electron states in the spacer results in an effective magnetic coupling between the magnetic layers. In both models, the oscillation period of the IEC is determined by the topology of the Fermi surface of the spacer. In particular, parallel regions of the Fermi surface connected by extremal spanning vectors perpendicular to the interface dominate the IEC due to their high density of electronic states. Multiple extremal spanning vectors lead to a superposition of oscillations with different periods. Both types of models imply the existence of a spatially oscillating electron spin density within the nonmagnetic spacer, but only the RKKY model gives a straightforward prediction for its spatial variation.
PACS numbers: 75.70.Ak, 75.70.Cn, 76.75.+i

While the IEC can be determined by experimental methods that probe the ferromagnetic layers, it is more difficult to measure directly the small induced polarization of the conduction electrons within the spacer. Especially, experimental techniques that probe the local induced polarization deep inside thick spacers and the attenuation of the polarization as a function of the distance from the magnetic interface have been lacking. Up to now, quantitative information about the polarization has been based on (i) techniques that measure an average polarization of the whole spacer [9] or (ii) methods that probe nonmagnetic overlayers on ferromagnetic substrates [10-12]. In the first case, the signal is dominated by the strong polarization of the first few monolayers due to the hybridization with the magnetic interface atoms. Local probe techniques such as Mössbauer spectroscopy, nuclear magnetic resonance, perturbed angular correlation, and nuclear orientation can provide valuable information on the local polarization [13-16], but due to their limited sensitivity they are generally restricted to the near interface region.

The muon spin rotation ( $\mu$ SR) technique offers a sufficiently high sensitivity to determine the induced polarization at large distances from the magnetic interface, since magnetic moments as small as $10^{-3}-10^{-4} \mu_{\mathrm{B}}$ can be detected. As a local probe, the muon senses the polarization of the surrounding conduction electrons via the magnetic hyperfine field at the interstitial lattice site. In a $\mu \mathrm{SR}$ experiment an ensemble of nearly $100 \%$ spinpolarized muons is implanted into the sample. The local magnetic field $B$, which is a sum of the hyperfine field and an externally applied field, causes a precession of the muon spin. The temporal evolution of the spin polarization $P(t)$ is monitored by the detection of the 
anisotropically emitted decay positrons. From this, the internal magnetic field distribution $p(B)$ can be deduced. The tunable low energy muon beam at the Paul Scherrer Institute, Switzerland, opens the possibility to use muons as radioactive microscopic magnetic probes that can be implanted at variable depth $(1-200 \mathrm{~nm})$ below the surface of any material [17].

In this Letter we report on the first application of $\mu \mathrm{SR}$ to the study of magnetic layered films. The low energy muon spin rotation measurements (LE- $\mu \mathrm{SR}$ ) were performed on an epitaxial $\mathrm{Fe} / \mathrm{Ag} / \mathrm{Fe}(001)$ trilayer. This system is an ideal case for study since high quality lattice matched epitaxial samples can be prepared and the $\mathrm{Ag}$ Fermi surface is well known from de Haas-van Alphen and cyclotron resonance experiments [18]. From our measurements we deduce the attenuation of the induced oscillatory electron spin polarization at large distances from the magnetic interface and show that the polarization decays much slower than expected.

The sample was grown by molecular beam epitaxy in an ultrahigh vacuum (UHV) system with a base pressure below $10^{-10}$ mbar and a pressure during the deposition in the low $10^{-10} \mathrm{hPa}$ range. The substrate was a $\mathrm{MgO}(001)$ plate, cleaved from a block prior to the introduction into the UHV system. After annealing in UHV for $1 \mathrm{~h}$ at $620^{\circ} \mathrm{C}$, it revealed a clean $\mathrm{MgO}(001)$ surface as checked by Auger-electron spectroscopy and low-energy electron diffraction (LEED). The sample was evaporated onto the substrate at a rate of about $0.5 \mathrm{~nm} / \mathrm{min}$ in a layer sequence with the nominal thicknesses of $1 \mathrm{~nm} \mathrm{Au} / 4 \mathrm{~nm}{ }^{57} \mathrm{Fe} / 20 \mathrm{~nm} \mathrm{Ag} / 4 \mathrm{~nm}{ }^{57} \mathrm{Fe} / \mathrm{MgO}$. The uppermost $\mathrm{Au}$ layer was added to protect the sample from oxidation. The layer thicknesses, with a typical uncertainty of $5 \%$, were controlled during evaporation by using a quartz microbalance. Rutherford backscattering and $\mathrm{x}$-ray diffraction measurements were performed, which confirmed the layer thicknesses. The epitaxial growth was monitored by means of LEED during preparation. Spot profile LEED analysis revealed $\geq 20 \mathrm{~nm}$-sized flat terraces, which were also confirmed by ex situ atomic force microscopy measurements for the uppermost Au layer. From ${ }^{57} \mathrm{Fe}$ conversion electron Mössbauer spectroscopy measurements, the diffusion of the $\mathrm{Fe}$ atoms into $\mathrm{Ag}$ is found to be smaller than 1.5 ML at each interface. A strong magnetic in-plane anisotropy was deduced from the Mössbauer emission line intensities. The longitudinal magneto-optical Kerr effect was applied to find the magnetic easy axis of the sample, which was the $\mathrm{Fe}$ [100]/MgO [110] direction.

The time differential LE- $\mu$ SR measurements were performed in external magnetic fields $B_{\text {ext }}=8.8 \mathrm{mT}$ applied perpendicular to the initial muon spin polarization $P(0)$ and parallel to the magnetic easy axis of the film. The sample was first magnetized at room temperature in a field of $B_{\text {ext }}=0.1 \mathrm{~T}$. In the remanent state ( $70 \%$ of saturation magnetization) it was then cooled to $20 \mathrm{~K}$. At this temperature muon diffusion after implantation can be neglected. Muons were implanted into the sample with an energy of $E=3 \mathrm{keV}$ giving a stopping distribution $n(x, E) \quad(x=$ distance from the first $\mathrm{Fe} / \mathrm{Ag}$ interface) which is centered in the $20 \mathrm{~nm} \mathrm{Ag}$ spacer (see inset of Fig. 1). The profile $n(x, E)$ has been calculated with the Monte Carlo code TRIM.SP [19] which gives very reliable results for $n(x, E)$ [20]. About $70 \%$ of the muons come to rest in Ag while the remainder stop in the boundary layers or do not hit the sample.

In Fig. 1 the time dependent muon decay asymmetry $A(t)$ is shown. The measured asymmetry is proportional to the polarization of the muon spin ensemble, $A(t)=$ $A_{0} P(t)$, where $A_{0}$ is the experimentally determined initial asymmetry. Muons stopped in the Fe layers and near the ferromagnetic interface, where a large induced polarization is expected, experience a wide distribution of large magnetic fields which leads to a very rapid depolarization. This fast depolarization cannot be observed in our LE- $\mu$ SR experiment and results in a reduction of the initial asymmetry from $A_{0}=0.245$ obtained for a pure Ag sample to $A_{0}=0.13$. From these values it follows that the $\mu$ SR measurement presented in Fig. 1 represents the time evolution of the spin polarization of muons thermalized within the inner $14 \mathrm{~nm}$ of the Ag layer. The LE- $\mu$ SR spectrum contains the information on the probability of the internal fields $p(B)$ experienced by the muon ensemble, which is related to $P(t)$ by

$$
P(t)=P(0) \int p(B) \cos \left(\gamma_{\mu} B t+\varphi\right) \mathrm{d} B
$$

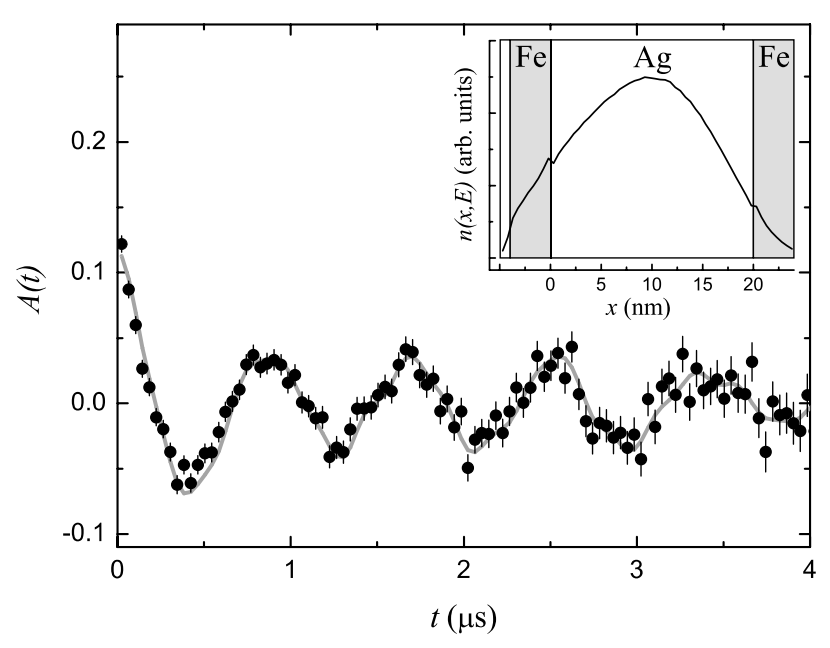

FIG. 1. LE- $\mu$ SR decay asymmetry as a function of time obtained at a temperature of $20 \mathrm{~K}$ in an external field of $B_{\text {ext }}=$ $8.8 \mathrm{mT}$. Only muons stopping in the inner $14 \mathrm{~nm}$ of the $20 \mathrm{~nm}$ $\mathrm{Ag}$ spacer contribute to $A(t)$. Muons thermalized in Fe or in interface regions of $\approx 3 \mathrm{~nm}$ sense too high magnetic fields to be detected with the LE- $\mu$ SR experiment. The solid line is a maximum entropy fit to the data using Eq. (1). Inset: Muon stopping profile for a muon implantation energy $E=3 \mathrm{keV}$, calculated with the Monte Carlo code TRIM.SP. 
where $\gamma_{\mu}=2 \pi \times 135.5 \mathrm{MHz} / \mathrm{T}$ is the gyromagnetic ratio of the muon and $\varphi$ is the angle between $P(0)$ and the first positron detector. Therefore, it is possible to derive the field distribution from a large portion of the spacer from one LE- $\mu$ SR measurement without the necessity to rely on several samples with different thicknesses. To obtain $p(B)$, the data have been analyzed in three different ways: (i) by fitting a distribution of internal fields to $A(t)$ in the time domain, (ii) by transforming $A(t)$ using a standard Fast Fourier algorithm, and (iii) by using a maximum entropy method [21]. All three methods give essentially the same $p(B)$. The field distribution obtained from the maximum entropy analysis is shown in Fig. 2.

The peak at $B_{\text {ext }}=8.8 \mathrm{mT}$ in the center of the field distribution is partially due to a background fraction of $10 \%$ of the muons that do not hit the sample. Local fields different from $B_{\text {ext }}$ are due to hyperfine interactions with the spatially oscillating electron spin polarization. The peaks seen in Fig. 2 reflect the higher probability density of $B$ at those depths in the layer where the oscillating $B$ is maximum or minimum. Thus, they directly reflect the variation of $B$ within the spacer layer. From the measured $B$ the induced local magnetization at the muon site $M_{\mathrm{loc}}=\left(B-B_{\mathrm{ext}}\right) V_{\mathrm{Ag}} / \mu_{0}$ (here, $M_{\mathrm{loc}}$ is expressed per

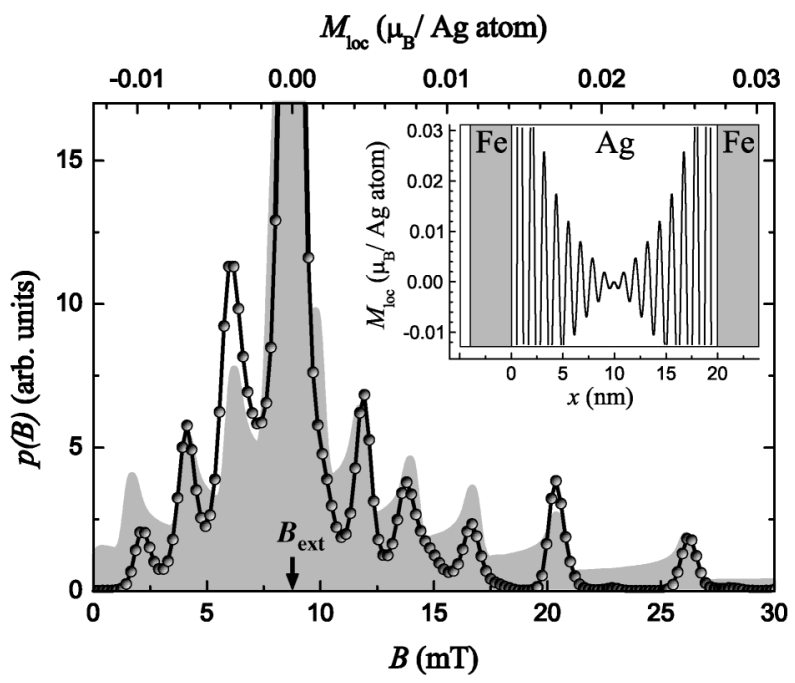

FIG. 2. Measured field distribution (points) inside the inner $14 \mathrm{~nm}$ of the $\mathrm{Ag}$ spacer at $20 \mathrm{~K}$ in an external field of $B_{\text {ext }}=$ $8.8 \mathrm{mT}$. The local field at the muon includes positive and negative hyperfine field contributions, which arise from the spatially oscillating polarization in the spacer. The measured fields can be directly converted into the local induced magnetization $M_{\text {loc }}$ (upper scale) at the muon sites at various depths within the layer. By comparison with a model distribution (shaded areas) it is possible to determine the spatial magnetic profile within the Ag spacer (inset). The model is described in the text and has been convoluted with a Gaussian of width $0.2 \mathrm{mT}$ to represent the finite frequency resolution of the $\mu \mathrm{SR}$ technique arising from the muon lifetime. volume of an $\mathrm{Ag}$ atom $V_{\mathrm{Ag}}$ ) can be calculated; see upper scale of Fig. 2.

The measured $p(B)$ is compared with a model field distribution obtained from a simple analytic description of $M_{\mathrm{loc}}(x)$ weighted with the muon stopping profile $n(x, E)$. The magnetization in the spacer is given by the superposition of the magnetic profiles induced by each of the two $\mathrm{Fe} / \mathrm{Ag}$ interfaces $\left[M_{\mathrm{loc}}(x)=M(x)+M(D-x)\right.$, where $x$ is the distance to the first interface and $D=$ $20 \mathrm{~nm}$ is the thickness of the $\mathrm{Ag}$ layer]. We assume $M(x)$ to vary in analogy to the asymptotic description of the IEC for large spacer layer thicknesses [5] as

$$
M(x)=\sum_{i} C_{i} x^{-\alpha_{i}} \sin \left(2 \pi x / \Lambda_{i}+\phi_{i}\right) .
$$

The sum is performed over contributions from extremal spanning vectors of the Ag Fermi surface, i.e., the spanning vectors at the belly and neck in the [001] direction in the first Brillouin zone. Electrons from these parts of the Fermi surface dominate the IEC [22], therefore the same behavior is expected for $M(x)$. The phases $\phi_{i}$ were fixed to the values given in [5]. The wavelengths $\Lambda_{\text {belly }}=$ $1.17 \mathrm{~nm}$ and $\Lambda_{\text {neck }}=0.485 \mathrm{~nm}$ were precisely measured with scanning electron microscopy with polarization analysis on wedged $\mathrm{Fe} / \mathrm{Ag} / \mathrm{Fe}$ samples [22]. These periods include the aliasing effect, which results from sampling the oscillations at discrete spacer thicknesses in steps of one monolayer. The LE- $\mu$ SR measurements sample $M_{\mathrm{loc}}(x)$ in the same manner since the octahedral interstitial muon sites in the fcc Ag lattice are separated by one monolayer along the sample normal. In this model, only the amplitude $C_{i}$ and the exponent $\alpha_{i}$ which describes the attenuation of the induced polarization are free fit parameters. Figure 2 shows the best fit to the measured $p(B)$. No contribution from the neck electron states is found. The measured $p(B)$ can be modeled taking into account only the long period oscillation from the belly of the Fermi surface. Moreover, it turns out that the induced polarization is of extreme long range nature $\left[\alpha_{\text {belly }}=0.8(1)\right]$. Hence, the electron spin polarization sensed by the muon at large distance $(x \geq 3 \mathrm{~nm})$ from the interfaces is much stronger than expected from the variation of the IEC strength with distance, which was found to be consistent with a $D^{-2}$ dependence for layer thicknesses $D \leq 3.7 \mathrm{~nm}$ [23]. From our data the amplitude of $M_{\text {loc }}$ slowly decreases from $2.6 \times 10^{-2} \mu_{\mathrm{B}} / \mathrm{Ag}$ at about $3 \mathrm{~nm}$ from the interface to $1.2 \times 10^{-3} \mu_{\mathrm{B}} / \mathrm{Ag}$ in the center of the spacer (see inset of Fig. 2).

It was shown for the $\mathrm{Fe} / \mathrm{Cu}$ system by numerical calculations using a first-principles Green's function formalism that a linear superposition of magnetic profiles of two independent interfaces gives a good first approximation of the moments in a paramagnetic spacer [24]. Small deviations of this magnetic profile are assigned to quantumwell states confined within the spacer and are taken into account by a small and nearly constant bias magnetization 
$M_{\mathrm{QW}}$. The fit to the LE- $\mu$ SR data shown in Fig. 2 has been improved by adding $M_{\mathrm{QW}}=-3 \times 10^{-4} \mu_{\mathrm{B}} / \mathrm{Ag}$ to $M_{\text {loc }}$. This bias, which is not predicted by RKKY-type models, has the correct sign and is of the right order of magnitude according to the calculated bias produced by quantum-well states in ferromagnetically aligned trilayers $[24,25]$.

The observation of the same periodicity $\Lambda_{\text {belly }}$ in the induced polarization $M(x)$ and in the IEC emphasizes their close relationship. However, the attenuation of the electron spin polarization, as seen by a pointlike local probe at large distances from the interface, is drastically smaller than the measured attenuation of the IEC strength in other systems (e.g., [26]). Equation (2) might be too crude an approximation for the spatial variation of $M_{\mathrm{loc}}$, but an analytic expression for the induced polarization including the quantum confinement of electrons within the spacer is not available for the $\mathrm{Fe} / \mathrm{Ag} / \mathrm{Fe}$ system. In Ref. [25] an asymptotic stationary phase formula for the oscillating spin density is derived and applied to $\mathrm{Co} / \mathrm{Cu} / \mathrm{Co}(001)$ trilayers. The spin polarization of the totally confined neck states is found to initially decay as $x^{-1}$ for the ferromagnetic configuration. It deviates from an $x^{-2}$ law for distances up to $20 \mathrm{~nm}$. Photoemission spectroscopy on $\mathrm{Fe} / \mathrm{Ag} / \mathrm{Fe}$ proves that the belly states are fully confined in this system [27]. Therefore, one may argue that their contribution also results in a slow decay of the polarization as was measured here. The short period oscillation from the neck states cannot be detected with LE- $\mu$ SR in our sample. It could be averaged out at the muon site by thickness variations of the spacer or roughness of the interfaces which are of the same order of magnitude as $\Lambda_{\text {neck }}$ [5]. Another explanation could be that the polarization of the neck states has already decayed for $x \geq 3 \mathrm{~nm}$ due to an attenuation with $\alpha_{\text {neck }} \approx 2$ as expected for these not fully confined states.

In conclusion, our data represent the first observation of the induced conduction electron spin polarization in the $\mathrm{Ag}$ spacer of a $\mathrm{Fe} / \mathrm{Ag} / \mathrm{Fe}$ trilayer for large distances from the interface $(x \geq 3 \mathrm{~nm})$. From the measured $p(B)$ sensed by the muon ensemble we were able to reconstruct the spatially oscillating polarization in the central $14 \mathrm{~nm}$ of the $20 \mathrm{~nm}$ thick Ag spacer. It can be well described using the long period oscillation originating from electron states from the belly of the Ag Fermi surface. A rather large amplitude of the oscillating polarization is found, ranging from $2.6 \times 10^{-2} \mu_{\mathrm{B}} / \mathrm{Ag}$ at about $3 \mathrm{~nm}$ from the interface to $1.2 \times 10^{-3} \mu_{\mathrm{B}} / \mathrm{Ag}$ in the center of the spacer. In the framework of the model used, this can be described by a $x^{-0.8(1)}$ dependence of the polarization indicating a different spatial attenuation than the IEC. This unexpected result cannot be accounted for by a simple RKKY approach. An explanation of this phenomenon may be found in theories including the full confinement of electron states within the spacer. We hope our results will stimulate detailed calculations.
We thank M. Pleines for her support during the LE- $\mu$ SR beam time, A. Brinck for the AFM, M. Doebeli for RBS measurements, and T. Riseman for providing the maximum entropy code. This work was performed at the Swiss Muon Source, Paul Scherrer Institut, Villigen, Switzerland. We acknowledge financial support from the UK EPSRC and the German BMBF.

*Electronic address: hubertus.luetkens@psi.ch

${ }^{\dagger}$ Present address: Zentralabteilung Technologie, FZ Jülich GmbH, D-52425 Jülich, Germany.

[1] S. S. P. Parkin, N. More, and K. P. Roche, Phys. Rev. Lett. 64, 2304 (1990).

[2] D. E. Bürgler, P. Grünberg, S. O. Demokritov, and M. T. Johnson, in Handbook of Magnetic Materials, edited by K. H. J. Buschow (Elsevier Science, Amsterdam, 2001), Vol. 13, pp. 1-85.

[3] Y. Yafet, Phys. Rev. B 36, 3948 (1987).

[4] R. Coehoorn, Phys. Rev. B 44, 9331 (1991).

[5] P. Bruno and C. Chappert, Phys. Rev. Lett. 67, 1602 (1991).

[6] D. M. Edwards, J. Mathon, R. B. Muniz, and M. S. Phan, Phys. Rev. Lett. 67, 493 (1991).

[7] P. Bruno, J. Phys. Condens. Matter 11, 9403 (1999).

[8] M. D. Stiles, J. Magn. Magn. Mater. 200, 322 (1999).

[9] M. G. Samant et al., Phys. Rev. Lett. 72, 1112 (1994).

[10] K. Koike, T. Furukawa, G. P. Cameron, and Y. Murayama, Phys. Rev. B 50, 4816 (1994).

[11] C. Carbone et al., Phys. Rev. Lett. 71, 2805 (1993).

[12] Y. Suzuki et al., Phys. Rev. Lett. 80, 5200 (1998).

[13] Y. Kobayashi, S. Nasu, T. Emoto, and T. Shinjo, Hyperfine Interact. 111, 129 (1998).

[14] Q.Y. Jin et al., Phys. Rev. Lett. 72, 768 (1994).

[15] B.-U. Runge et al., Phys. Rev. Lett. 79, 3054 (1997).

[16] T. Phalet et al., Phys. Rev. Lett. 86, 902 (2001).

[17] E. Morenzoni et al., Phys. Rev. Lett. 72, 2793 (1994).

[18] M. R. Halse, Philos. Trans. R. Soc. London, Ser. A 265, 507 (1969).

[19] W. Eckstein, Computer Simulation of Ion-Solid Interactions (Springer -Verlag, Berlin, 1991).

[20] E. Morenzoni et al., Nucl. Instrum. Methods Phys. Res., Sect. B 192, 254 (2002).

[21] N. Wu, in The Maximum Entropy Method, Springer Series in Information Science Vol. 32, edited by T.S. Huang, T. Kohonen, and M. R. Schroeder (SpringerVerlag, Berlin, 1997).

[22] J. Unguris, R. J. Celotta, and D. T. Pierce, J. Magn. Magn. Mater. 127, 205 (1993).

[23] Q. Leng et al., J. Magn. Magn. Mater. 126, 367 (1993).

[24] A. M. N. Niklasson, S. Mirbt, H. L. Skriver, and B. Johansson, Phys. Rev. B 53, 8509 (1996).

[25] J. Mathon, A. Umerski, M. Villeret, and R. B. Muniz, Phys. Rev. B 59, 6344 (1999).

[26] J. Unguris, R. J. Celotta, and D. T. Pierce, Phys. Rev. Lett. 79, 2734 (1997).

[27] J. E. Ortega and F. J. Himpsel, Phys. Rev. Lett. 69, 844 (1992). 